\title{
Effects of Shenmai injection and its bioactive components following ischemia/reperfusion in cardiomyocytes
}

\author{
LI-FANG YE ${ }^{1}$, YA-RU ZHENG ${ }^{1}$ and LI-HONG WANG ${ }^{2}$ \\ ${ }^{1}$ Department of Cardiology, First Affiliated Hospital, Medical College of Zhejiang University, Hangzhou, Zhejiang 310003; \\ ${ }^{2}$ Department of Cardiology, People's Hospital of Zhejiang Province, \\ Hangzhou, Zhejiang 310014, P.R. China
}

Received December 22, 2014; Accepted June 24, 2015

DOI: $10.3892 / \mathrm{etm} .2015 .2662$

\begin{abstract}
The aim of the present study was to determine whether the myocardial protective function of Shenmai injection (SM) during ischemia/reperfusion (I/R) is attributable to its regulation of intracellular calcium $\left(\mathrm{Ca}^{2+}\right)$ and phospholamban (PLB) levels. Cultured neonatal Sprague Dawley rat cardiomyocytes were used to compare the effects of normoxia, total saponins of Panax ginseng (TSPG), ginsenoside Rg1 (Rg1) and SM treatments in rat myocardial cells following $\mathrm{I} / \mathrm{R}$. For each of these treatment groups, the mRNA and protein levels of PLB and the sarco/endoplasmic reticulum $\mathrm{Ca}^{2+}$ ATPase (SERCA) were evaluated, in addition to the cytoplasmic $\mathrm{Ca}^{2+}$ concentration $\left[\mathrm{Ca}^{2+}\right]_{i}$ and the rate of apoptosis. The results indicated that I/R markedly decreased phosphorylated PLB and SERCA expression and that SM was able to mitigate this effect, while TPSG and Rg1 were not. Furthermore, SM appeared to prevent aberrant apoptosis and restore the depleted $\left[\mathrm{Ca}^{2+}\right]_{i}$ resulting from I/R. The protective efficacy of SM against heart disease following I/R may, therefore, be due in part to its effect on intracellular $\mathrm{Ca}^{2+}$ homeostasis. SM may exert its protective effects by relieving PLB inhibition, and the pharmacodynamic actions of SM appear to be significantly more effective than those of its bioreactive components, TPSG and Rgl.
\end{abstract}

\section{Introduction}

Human ischemic heart disease is the cause of $13.2 \%$ of all mortality worldwide (1). The upregulation of apoptosis and cardiac systolic dysfunction in diseased heart tissue are considered to contribute significantly to disease development and ultimate heart failure (2). Furthermore, a previous study

Correspondence to: Professor Li-Hong Wang, Department of Cardiology, People's Hospital of Zhejiang Province, 158 Shangtang Road, Hangzhou, Zhejiang 310014, P.R. China

E-mail: wanglhnew@126.com

Key words: Shenmai injection, ischemia/reperfusion, total saponins of Panax ginseng, phospholamban, ginsenoside Rg1, intracellular calcium suggested that the maintenance of intracellular calcium concentration $\left[\mathrm{Ca}^{2+}\right]_{\mathrm{i}}$ is critical for normal myocardial function (3). The major proteins responsible for maintaining intracellular $\mathrm{Ca}^{2+}$ homeostasis throughout excitation-contraction cycling in cardiomyocytes include sarco/endoplasmic reticulum $\mathrm{Ca}^{2+}$-ATPase (SERCA) and the SERCA regulatory protein phospholamban (PLB) (4). In its dephosphorylated state, PLB inhibits the uptake of $\mathrm{Ca}^{2+}$ by SERCA. By contrast, PLB phosphorylation by cAMP-dependent protein kinase A (PKA) at Ser16 and by $\mathrm{Ca}^{2+}$-calmodulin-dependent protein kinase II at Thr17 relieves this inhibitory influence on SERCA and thereby increases $\mathrm{Ca}^{2+}$ uptake by the sarcoplasmic reticulum (SR) (5). The phosphorylation of PLB, primarily by PKA at Ser16, is known to be associated with enhanced critical left ventricular functions, such as contractility and relaxation (6). The results of our previous studies showed that the induction of acute myocardial infarction in rats resulted in a significant downregulation of SERCA mRNA and protein expression and, conversely, caused a significant upregulation of PLB mRNA and protein expression $(7,8)$. We therefore hypothesize that aberrant cardiac contractile function partially contributes to these observed effects, but it would be useful to determine the mechanism used by myocardial cells to stabilize the $\left[\mathrm{Ca}^{2+}\right]_{\mathrm{i}}$. In addition, it would be beneficial to investigate whether the regulation of PLB phosphorylation is sufficient to reduce cell apoptosis and improve cardiac systolic function. Performing these investigations would likely provide crucial insight into methods for improving the treatment of coronary heart disease.

Shenmai injection (SM), derived from the shendong drink described in the Zheng Yin Mai Zhi (which translates as 'Pattern, Cause, Pulse and Treatment') by Qin Changyu of the Ming dynasty (9), has been widely applied in recent years to treat qi-yin (10-14). The term qi-yin derives from the word 'qi', which, according to Traditional Chinese Medicine theory, is defined as the basic energy that maintains life activities. 'Yin' and 'yang' are considered to be the two opposing principles in nature, with 'yin' representing the feminine and negative and 'yang' representing the masculine and positive. A 'qi-yin' deficiency is associated with coronary heart disease, chronic pulmonary heart disease and viral myocarditis, in addition to heart and respiratory failure (15). According to Traditional Chinese Medicine theory, SM benefits qi, prevents exhaustion, nourishes yin and replenishes bodily fluids, while demonstrating 
protective effects against adverse drug reactions (16). SM is extracted from red ginseng and the ophiopogon root. Ginsenosides, the primary bioactive components of ginseng, are known to scavenge oxygen free radicals $(17,18)$, block $\mathrm{Ca}^{2+}$ channels $(17,19)$ and reduce the ischemia/reperfusion $(\mathrm{I} / \mathrm{R})$ injury associated with cardiovascular and cerebrovascular diseases (20-24). Previous studies have indicated that ginsenoside Rg1 protects cardiomyocytes under hypoxic conditions by reducing intracellular $\mathrm{Ca}^{2+}$ overload (24-26). Treatment with the total saponins of Panax ginseng (TSPG), i.e. the total quantity of ginsenosides extracted from ginseng, can increase cardiac SR $\mathrm{Ca}^{2+}$ levels, reduce mitochondrial $\mathrm{Ca}^{2+}$ levels and increase mitochondrial calcium pump activity $(22,23)$. Furthermore, $\mathrm{SM}$ is able to mitigate apoptosis and $\mathrm{Ca}^{2+}$ influx in neurocytes following hypoxia-reoxygenation (21). It remains unclear, however, whether the myocardial protective effect of SM injection during I/R functions by maintaining the $\left[\mathrm{Ca}^{2+}\right]$ ${ }_{i}$ via the regulation of PLB phosphorylation. An aim of the present study was to elucidate the mechanism underlying PLB involvement in each of these processes.

This study aimed to determine whether the myocardial protection from SM injection during I/R is associated with the maintenance of the $\left[\mathrm{Ca}^{2+}\right]_{\mathrm{i}}$ through the relief of PLB inhibition. In addition, a further aim was to determine whether the pharmacodynamic activity of SM injection is superior to that of the pure ginseng saponins compounds.

\section{Materials and methods}

Chemicals and reagents. SM was purchased from Chia Tai Qingchunbao Pharmaceutical Co., Ltd. (Hangzhou, China). Ginsenoside Rg1 (purity, >99\%) was purchased from the Chinese National Institute for the Control of Pharmaceutical and Biological Products (Beijing, China). TSPG (purity, $>80 \%$ ) was purchased from Shanghai Winherb Medical Technology Co., Ltd. (Shanghai, China). Dulbecco's modified Eagle's medium (DMEM), fetal bovine serum (FBS) and TRIzol ${ }^{\circledR}$ reagent were obtained from Invitrogen Life Technologies (Carlsbad, CA, USA). Rabbit anti-PLB antibodies (\#05-205) and ECL-Plus chemiluminescent substrate were purchased from EMD Millipore (Billerica, MA, USA). Mouse anti-phosphorylated PLB (p-PLB; phosphorylated at Thr 17 and Ser 16; \#8496) and rabbit anti-SERCA antibodies (\#9580) were purchased from Cell Signaling Technology, Inc. (Danvers, MA, USA). Mouse anti- $\beta$-actin (\#CW0096) and horseradish peroxidase-labeled $\operatorname{IgG}$ secondary antibody were obtained from Beijing ComWin BioTech Co., Ltd. (Beijing, China). The PrimeScript ${ }^{\mathrm{TM}}$ RT reagent kit and $S Y B R^{\circledR}$ Premix Ex Taq ${ }^{\mathrm{TM}}$ II were from Takara Bio, Inc. (Otsu, Japan). Primers for the reverse transcription-quantitative polymerase chain reaction (RT-qPCR) were purchased from Sangon Biotech Co., Ltd. (Shanghai, China). The Annexin-V/propidium iodide (PI) Assay kit was purchased from BD Biosciences (Franklin Lakes, NJ, USA). Fluo-4-AM was purchased from Life Technologies.

Animals. The study was designed and all protocols involving animals were conducted according to the Guide for the Care and Use of Laboratory Animals published by the U.S. National Institutes of Health. In addition, the animal protocol utilized in this study was approved by the Ethics Committee of the Medical College of Zhejiang University (Hangzhou, China).

Primary cardiomyocyte culture. Cardiomyocytes were isolated from the hearts of neonatal Sprague Dawley rats that were $\leq 3$ days old (SLAC Laboratory Animal Co., Ltd., Shanghai, China), and the isolated cells were cultured as described previously with minor modifications (27). To obtain the cardiomyocytes, heart tissue was digested with $0.1 \%$ collagenase type II (Invitrogen Life Technologies) and $0.125 \%$ pancreatin (Sigma-Aldrich, St. Louis, MO, USA) for $8 \mathrm{~min}$ at $37^{\circ} \mathrm{C}$. Following centrifugation, the supernatant was discarded and the cell pellets were resuspended in culture media containing $10 \%$ FBS. These steps were repeated until the hearts were completely digested. Cells were pre-plated for $90 \mathrm{~min}$ to allow fibroblasts to attach and to yield a purer cardiomyocyte population. The cardiomyocytes were maintained in DMEM containing 10\% FBS, $0.1 \mathrm{mM}$ 5-bromo-2'-deoxyuridine (Invitrogen Life Technologies) and $100 \mathrm{IU} / \mathrm{ml} 0.3 \%$ penicillin-streptomycin to inhibit the growth of other cell types. Following 3 days in culture, the cardiomyocytes were subjected to subsequent experimentation.

The cardiomyocytes were divided into the following eight groups: Normoxia (N), TSPG-treated normoxia (N + TSPG), Rg1-treated normoxia ( $+R g 1)$, SM-treated normoxia $(\mathrm{N}+\mathrm{SM}), \mathrm{I} / \mathrm{R}$, TSPG-treated I/R (I/R + TSPG), Rg1-treated $\mathrm{I} / \mathrm{R}(\mathrm{I} / \mathrm{R}+\mathrm{Rg} 1)$ and SM-treated I/R (I/R + SM).

Hypoxia/reoxygenation treatment protocol. Cardiomyocytes were pretreated with TSPG (1.5 mg/l), Rg1 (0.325 mg/l) or SM $(5 \mathrm{ml} / \mathrm{l})$ for $24 \mathrm{~h}$. Cultured cardiomyocytes were subsequently washed with Hank's balanced salt solution (HBSS), containing $5 \mathrm{mM}$ HEPES, $137 \mathrm{mM} \mathrm{NaCl}, 4 \mathrm{mM} \mathrm{KCl}, 1 \mathrm{mM} \mathrm{MgCl}{ }_{2}$ and $1.5 \mathrm{mM} \mathrm{CaCl}_{2}$ (pH 7.2), and then incubated in glucose-free DMEM. Hypoxia was used to mimic the in vivo condition of myocardial ischemia. To induce hypoxia, the cells were placed in an incubator at $37^{\circ} \mathrm{C}$, and then $\mathrm{N}_{2}(95 \%)$ and $5 \% \mathrm{CO}_{2}$ were flushed into the incubator to lower the oxygen concentration to $1 \%$. Following $4 \mathrm{~h}$ of exposure to the hypoxic conditions, the cells were subjected to reoxygenation by exchanging the media with DMEM supplemented with $4.5 \mathrm{~g} / 1$ glucose. Cells were then incubated under normoxic conditions for $1 \mathrm{~h}$.

RNA analysis. Total RNA from the cardiomyocytes was isolated using TRIzol reagent according to the manufacturer's instructions. Total RNA concentration was determined photometrically using a wavelength of $260 \mathrm{~nm}$. RNA samples were stored at $-80^{\circ} \mathrm{C}$. For the RT-qPCR, total RNA was transcribed using the PrimeScript RT reagent kit. The PCR reaction conditions were as follows: $95^{\circ} \mathrm{C}$ for $30 \mathrm{sec}$, then 40 cycles of $5 \mathrm{sec}$ at $95^{\circ} \mathrm{C}$ and $34 \mathrm{sec}$ at $60^{\circ} \mathrm{C}$. Individual samples of $100 \mathrm{ng}$ cDNA were amplified using SYBR Premix Ex Taq II, utilizing gene-specific primers in an ABI PRISM ${ }^{\circledR} 7500$ Fast Sequence Detection System (Applied Biosystems, Foster City, CA, USA). Standard curves were performed in duplicate with serially diluted cDNA synthesized from neonatal rat heart tissue (1.5-50 ng) to determine the PCR efficiency, which yielded similar results in all groups. SERCA and PLB mRNA expression levels were evaluated as a ratio of SERCA/PLB. Quantification was performed using the standard curve and $2^{-\Delta \Delta \mathrm{Ct}}$ methods. 
A

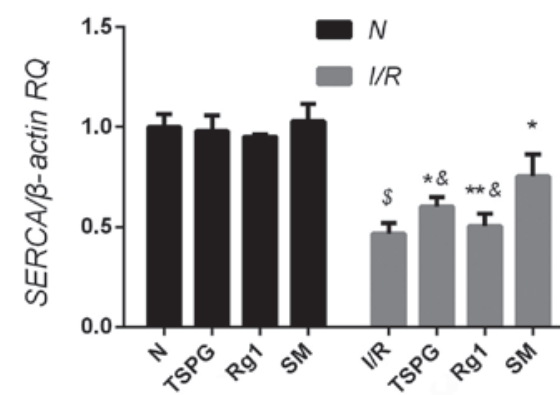

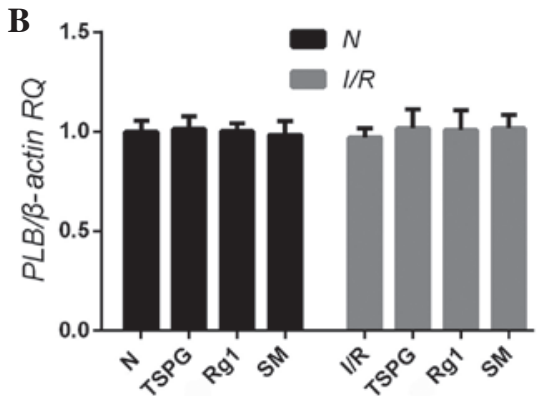

Figure 1. Effects of TSPG, Rg1 and SM on SERCA and PLB mRNA expression following I/R in cardiomyocytes. (A) SERCA and (B) PLB mRNA expression levels. Data are expressed as the mean \pm standard deviation $(\mathrm{n}=9) .{ }^{\$} \mathrm{P}<0.01$ vs. $\mathrm{N} ;{ }^{\&} \mathrm{P}<0.01$ vs. I/R + SM; ${ }^{*}<0.01$ vs. I/R; ${ }^{* *} \mathrm{P}<0.01$ vs. I/R+TSPG. SERCA, sarco/endoplasmic reticulum $\mathrm{Ca}^{2+}$ ATPase; PLB, phospholamban; N, normoxia; RQ, relative quantification; I/R, ischemia/reperfusion; TSPG, total saponins of Panax ginseng; Rg1, ginsenoside Rg1; SM, Shenmai injection.

The primers used for PCR analysis were as follows: SERCA2a forward, 5'-AAGCAGTTCATCCGCTACCT-3' and reverse, 5'-AGACCATCCGTCACCAGATT-3'; PLB forward, 5'-TACCTTACTCGCTCGGCTATC-3' and reverse, 5'-TAC CTTACTCGCTCGGCTATC-3'; and $\beta$-actin forward, 5'-GGA GATTACTGCCCTGGCTCCTA-3' and reverse, 5'-GACTCA TCGTACTCCTGCTTGCTG-3'.

Western blot analysis. Isolated cardiomyoctes were lysed in radioimmunoprecipitation assay buffer $(50 \mathrm{mM}$ Tris- $\mathrm{HCl}$ pH 7.4, $150 \mathrm{mM} \mathrm{NaCl}, 1 \% \mathrm{NP}-40,10.5 \%$ sodium deoxycholate, $5 \%$ sodium dodecyl sulfate and $1 \mathrm{mM}$ phenylmethyl sulfonyl-fluoride). The total protein concentration was quantified using a bicinchoninic protein quantity assay kit (Thermo Fisher Scientific, Inc., Waltham, MA, USA) and equal amounts of protein were loaded onto $12 \%$ sodium dodecyl sulfate-polyacrylamide gels. Following separation by electrophoresis, the proteins were electrotransferred onto polyvinylidene difluoride membranes. Non-specific binding sites were blocked by incubating with $5 \%$ skimmed milk in Tris-buffered saline containing $0.05 \%$ Tween-20 at room temperature for $1 \mathrm{~h}$. The membranes were probed using rabbit anti-PLB $(1: 1,000)$, mouse anti-p-PLB $(1: 1,000)$, rabbit anti-SERCA2a $(1: 1,000)$ and mouse anti- $\beta$-actin $(1: 1,000)$ primary antibodies overnight at $4{ }^{\circ} \mathrm{C}$. The membranes were subsequently probed with horseradish peroxidase-labeled anti-rabbit $\operatorname{IgG}$ secondary antibody $(1: 10,000)$ at room temperature for $1 \mathrm{~h}$. The immunoreactive bands were visualized using ECL-Plus reagent. Signal intensities of each band were analyzed using Quantity One ${ }^{\circledR}$ software, version 4.6.2 (Bio-Rad Laboratories, Inc., Berkeley, CA, USA), and the relative protein levels were calculated by comparing with the $\beta$-actin loading control.

Annexin V/PI assay. Briefly, cardiomyocytes were collected, washed with $\mathrm{Ca}^{2+}$-free phosphate-buffered saline, resuspended in binding buffer and incubated with $5 \mu \mathrm{l}$ Annexin V and PI at room temperature in the dark for $15 \mathrm{~min}$. The cardiomyocytes were then analyzed using a flow cytometer (FC500MCL; Beckman Coulter, Brea, CA, USA).

Measurement of $\left[\mathrm{Ca}^{2+}\right]_{i}$. After $1 \mathrm{~h}$ of reoxygenation, $\left[\mathrm{Ca}^{2+}\right]_{\mathrm{i}}$ measurements were conducted using the $\mathrm{Ca}^{2+}$-sensitive fluorescent probe fluo-4 AM. Cardiomyocytes were incubated in six-well plates with $5 \mu \mathrm{M}$ fluo- $4 \mathrm{AM}$ for $30 \mathrm{~min}$ at $37^{\circ} \mathrm{C}$. The cells were washed with HBSS three times. Fluorescence levels were measured using flow cytometry, with excitation at $484 \mathrm{~nm}$ and emission at $516 \mathrm{~nm}$.

Statistical analysis. Statistical analyses were performed using SPSS software, version 19.0 (IBM SPSS, Armonk, NY, USA). Statistical significance was detected using one-way analysis of variance and Student's $t$-tests. $\mathrm{P}<0.05$ was considered to indicate a statistically significant difference.

\section{Results}

Effects of TSPG, Rg1 and SM on SERCA and PLB mRNA expression levels. In order to determine whether TSPG, Rg1 or SM treatment was able to affect SERCA and PLB mRNA expression, rat myocardial cells were subjected to $I / R$, and the resulting mRNA levels were compared with those of the normoxic cells. No significant difference in the PLB mRNA levels was detected between the I/R group and the untreated controls $(\mathrm{P}>0.05)$; however, the SERCA mRNA levels were found to be significantly decreased in the $\mathrm{I} / \mathrm{R}$ group $(\mathrm{P}<0.01)$. Treatment of the I/R cells with TSPG (I/R + TPSG) or SM $(\mathrm{I} / \mathrm{R}+\mathrm{SM})$ resulted in a significant upregulation of SERCA mRNA levels, with SM treatment showing the most marked effect $(\mathrm{P}<0.01)$. Notably, this difference in mRNA expression was not evident in the $\mathrm{I} / \mathrm{R}+\mathrm{Rg} 1$ group $(\mathrm{P}>0.05)$ (Fig. 1).

Effects of TSPG, RgI and SM on SERCA, PLB and $p-P L B$ protein levels. In addition to the mRNA analysis, this study aimed to determine whether TSPG, Rg1 or SM treatment was able to affect SERCA, PLB and/or p-PLB protein expression. Following I/R treatment, the PLB protein levels in the I/R treatment group were compared with those in the $\mathrm{N}$ group, and no statistically significant difference was detected $(\mathrm{P}>0.05)$. Conversely, the protein levels of p-PLB and the p-PLB/PLB ratio were significantly reduced in the $\mathrm{I} / \mathrm{R}$ group compared with those in the $\mathrm{N}$ group (both $\mathrm{P}<0.01$ ); however, the $\mathrm{I} / \mathrm{R}+\mathrm{SM}$ group exhibited a significant increase in p-PLB protein levels $(\mathrm{P}<0.01)$, in addition to an increase in the $\mathrm{p}-\mathrm{PLB} / \mathrm{PLB}$ expression ratio, compared with the $\mathrm{I} / \mathrm{R}$ group $(\mathrm{P}<0.01)$. Notably, the two other drug administration groups (TSPG and Rg1) showed no significant alterations in $\mathrm{p}-\mathrm{PLB}$ protein expression $(\mathrm{P}>0.05)$ or $\mathrm{p}-\mathrm{PLB} / \mathrm{PLB}$ ratio $(\mathrm{P}>0.05)$. 


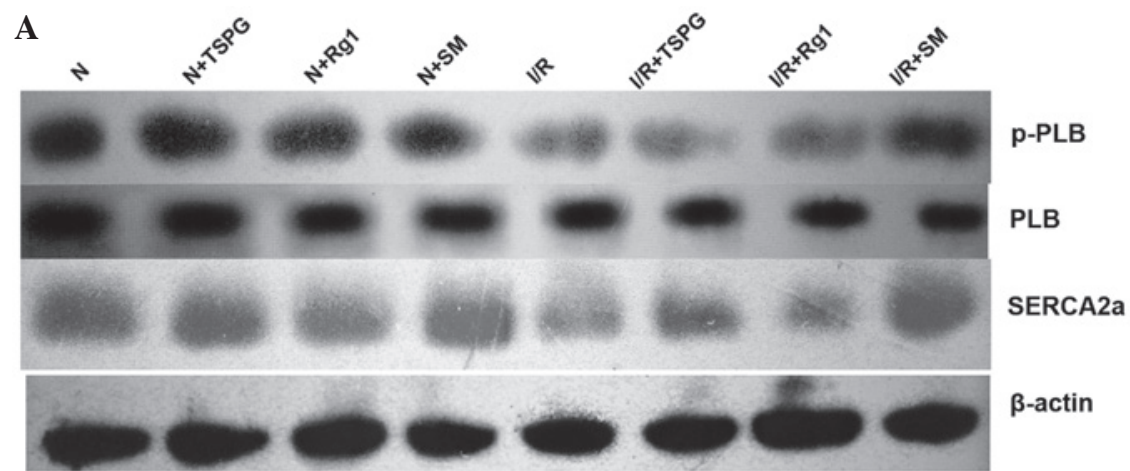

B

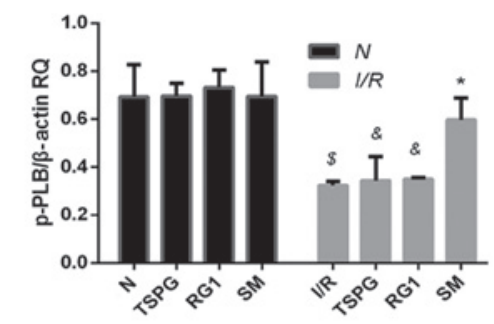

D

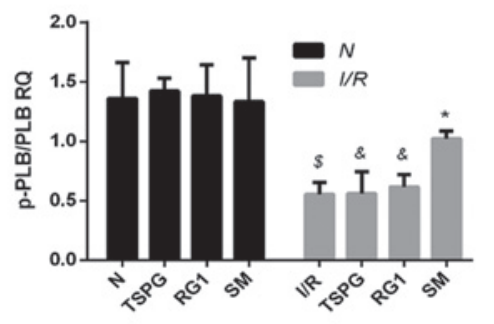

C

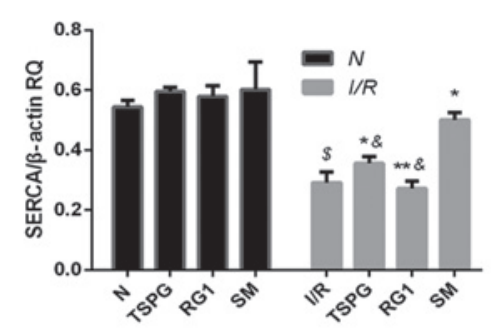

$\mathbf{E}$

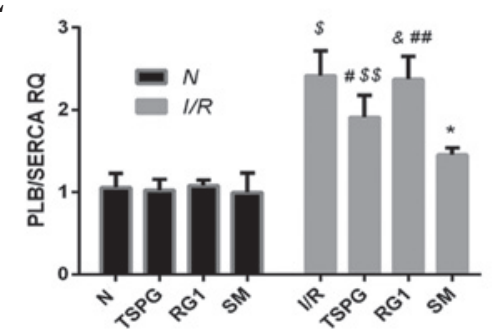

Figure 2. Effects of TSPG, Rg1 and SM on SERCA, p-PLB, p-PLB/PLB and PLB/SERCA levels following I/R in cardiomyocytes. (A) Western blot analysis of SERCA, p-PLB, PLB and $\beta$-actin levels. (B-E) Quantification of (B) p-PLB levels, (C) SERCA levels, (D) p-PLB/PLB ratio and (E) PLB/SERCA ratio. Data are expressed as the mean \pm standard deviation $(\mathrm{n}=4) .{ }^{\$} \mathrm{P}<0.01$ vs. $\mathrm{N} ;{ }^{\circledR} \mathrm{P}<0.01$ and ${ }^{\$ S} \mathrm{P}<0.05$ vs. I/R $+\mathrm{SM} ;{ }^{*} \mathrm{P}<0.01$ and ${ }^{\#} \mathrm{P}<0.05$ vs. I/R; ${ }^{* *} \mathrm{P}<0.01$ and ${ }^{\# \#} \mathrm{P}<0.05$ vs. I/R + TSPG. N, normoxia; TSPG, total saponins of Panax ginseng; Rg1, ginsenoside Rg1; SM, Shenmai injection; I/R, ischemia/reperfusion; PLB, phospholamban; p-PLB, phosphorylated-PLB; SERCA, sarco/endoplasmic reticulum $\mathrm{Ca}^{2+} \mathrm{ATPase}$; RQ, relative quantification.

The comparison of SERCA protein levels between the $\mathrm{N}$ and $\mathrm{I} / \mathrm{R}$ treatment groups revealed a significant reduction following I/R treatment $(\mathrm{P}<0.01)$. Conversely, the SERCA protein expression levels were significantly elevated in the $\mathrm{I} / \mathrm{R}+\mathrm{SM}$ and $\mathrm{I} / \mathrm{R}+\mathrm{TSPG}$ groups compared with the levels in the $\mathrm{I} / \mathrm{R}$ group $(\mathrm{P}<0.01)$, whereas the SERCA protein expression in the $\mathrm{I} / \mathrm{R}$ and $\mathrm{I} / \mathrm{R}+\mathrm{Rg} 1$ groups showed no significant difference $(\mathrm{P}>0.05)$. The $\mathrm{PLB} / \mathrm{SERCA}$ ratio was significantly higher in the I/R group compared with that in the $\mathrm{N}$ group $(\mathrm{P}<0.01)$, while this ratio was significantly decreased in the $\mathrm{I} / \mathrm{R}+\mathrm{SM}(\mathrm{P}<0.01)$ and $\mathrm{I} / \mathrm{R}+\mathrm{TSPG}(\mathrm{P}<0.05)$ groups. The $\mathrm{I} / \mathrm{R}+\mathrm{Rg} 1$ group exhibited a modest but insignificant reduction in the PLB/SERCA ratio $(\mathrm{P}>0.05)$. Notably, the $\mathrm{I} / \mathrm{R}+\mathrm{SM}$ group showed the most marked increase in SERCA protein levels and the most marked reduction in the PLB/SERCA ratio compared with the I/R + TSPG group $(\mathrm{P}<0.05 / 0.01)$ (Fig. 2).

Effects of TSPG, RgI and SM on cardiomyocyte apoptosis following I/R injury. In order to examine the effects of TSPG, Rg1 and SM treatment on I/R-induced apoptosis, the apoptosis rates were determined using flow cytometry. As shown in Fig. 3, I/R treatment markedly increased the apoptosis rates $(\mathrm{P}<0.01)$; however, this effect was attenuated with $\mathrm{SM}$ treatment $(\mathrm{P}<0.05)$. In addition, the $\mathrm{I} / \mathrm{R}+\mathrm{Rg} 1$ group showed a modest, insignificant reduction in apoptosis $(\mathrm{P}>0.05)$, and the $\mathrm{I} / \mathrm{R}+\mathrm{TSPG}$ group exhibited a greater but also insignificant reduction in apoptosis $(\mathrm{P}>0.05)$ (Fig. 3).

Effects of TSPG, RgI and SM on $\left[\mathrm{Ca}^{2+}\right]_{i}$ in cardiomyocytes following I/R injury. As shown in Fig. 4,I/R markedly increased the $\left[\mathrm{Ca}^{2+}\right]_{\mathrm{i}}(\mathrm{P}<0.01)$; however, this increase was suppressed by the SM $(\mathrm{P}<0.01)$ and TSPG $(\mathrm{P}<0.05)$ treatments. The I/R + Rg1 group exhibited a modest but insignificant decrease in $\left[\mathrm{Ca}^{2+}\right]_{\mathrm{i}}$ compared with the $\mathrm{I} / \mathrm{R}$ group $(\mathrm{P}>0.05)$. The $\mathrm{I} / \mathrm{R}+\mathrm{SM}$ group showed the greatest decrease in $\left[\mathrm{Ca}^{2+}\right]_{\mathrm{i}}$ following $\mathrm{I} / \mathrm{R}$ treatment $(\mathrm{P}<0.01)$ (Fig. 4).

\section{Discussion}

In the present study, the mechanism underlying the myocardial protective effect of SM following I/R was investigated. It was demonstrated that exposure to I/R resulted in significant alterations in a variety of functional indices for cardiac function, including a disruption in $\mathrm{Ca}^{2+}$ transport within heart myocardial cells. In accordance with a previous study, the apoptosis rate and $\left[\mathrm{Ca}^{2+}\right]_{i}$ of cardiomyocytes increased signifi- 

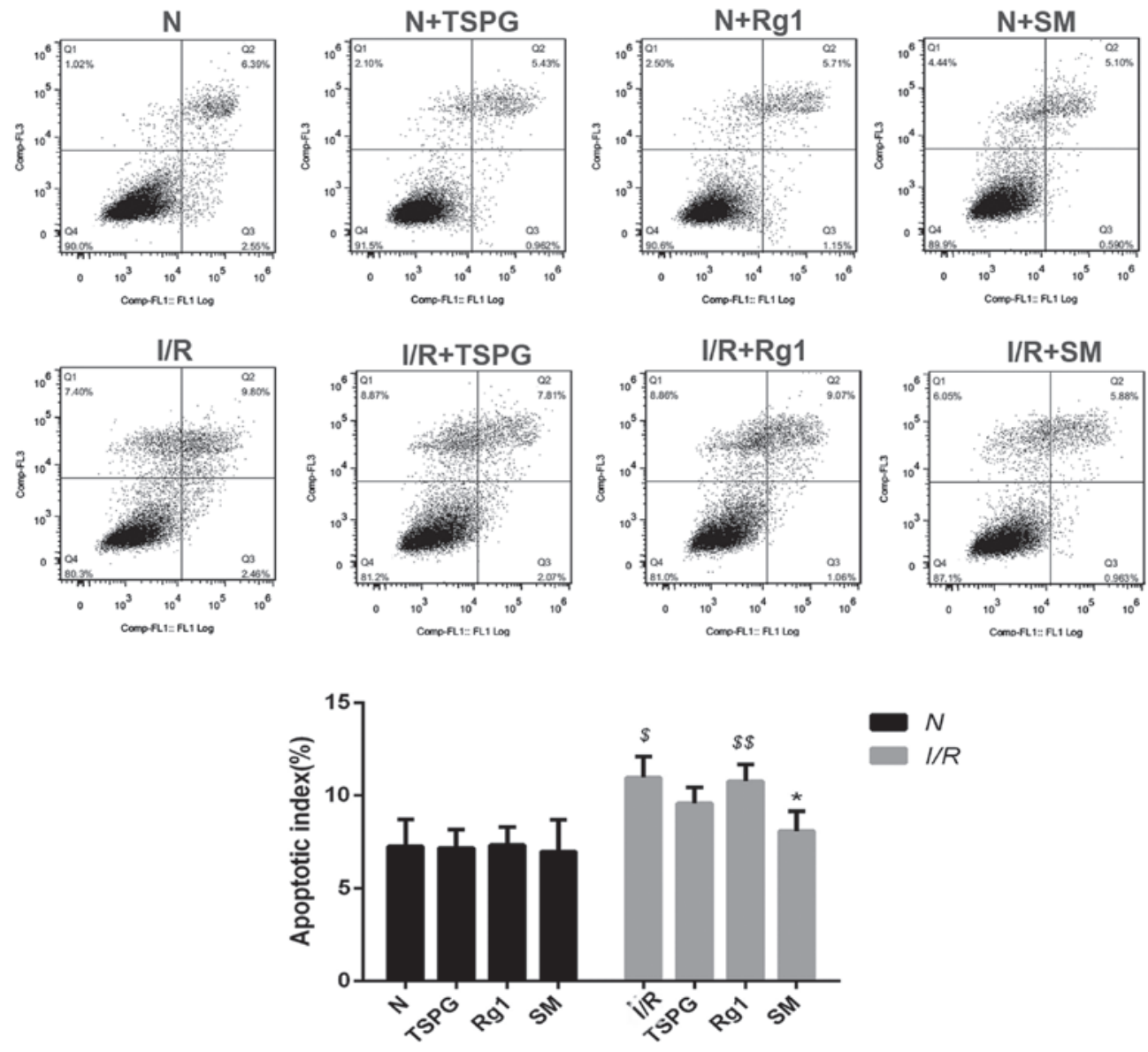

Figure 3. Effects of TSPG, Rg1 and SM on I/R-induced cardiomyocyte apoptosis. Cardiomyocytes in regions Q3 and Q2 represent early- and late-apoptotic cardiomyocytes, respectively. Data are expressed as the mean \pm standard deviation $(n=3) .{ }^{\$} \mathrm{P}<0.01 \mathrm{vs} . \mathrm{N} ;{ }^{\$ \$} \mathrm{P}<0.05$ vs. I/R $+\mathrm{SM}$; ${ }^{*} \mathrm{P}<0.01 \mathrm{vs}$. I/R. N, normoxia; TSPG, total saponins of Panax ginseng; Rg1, ginsenoside Rg1; SM, Shenmai injection; I/R, ischemia/reperfusion.
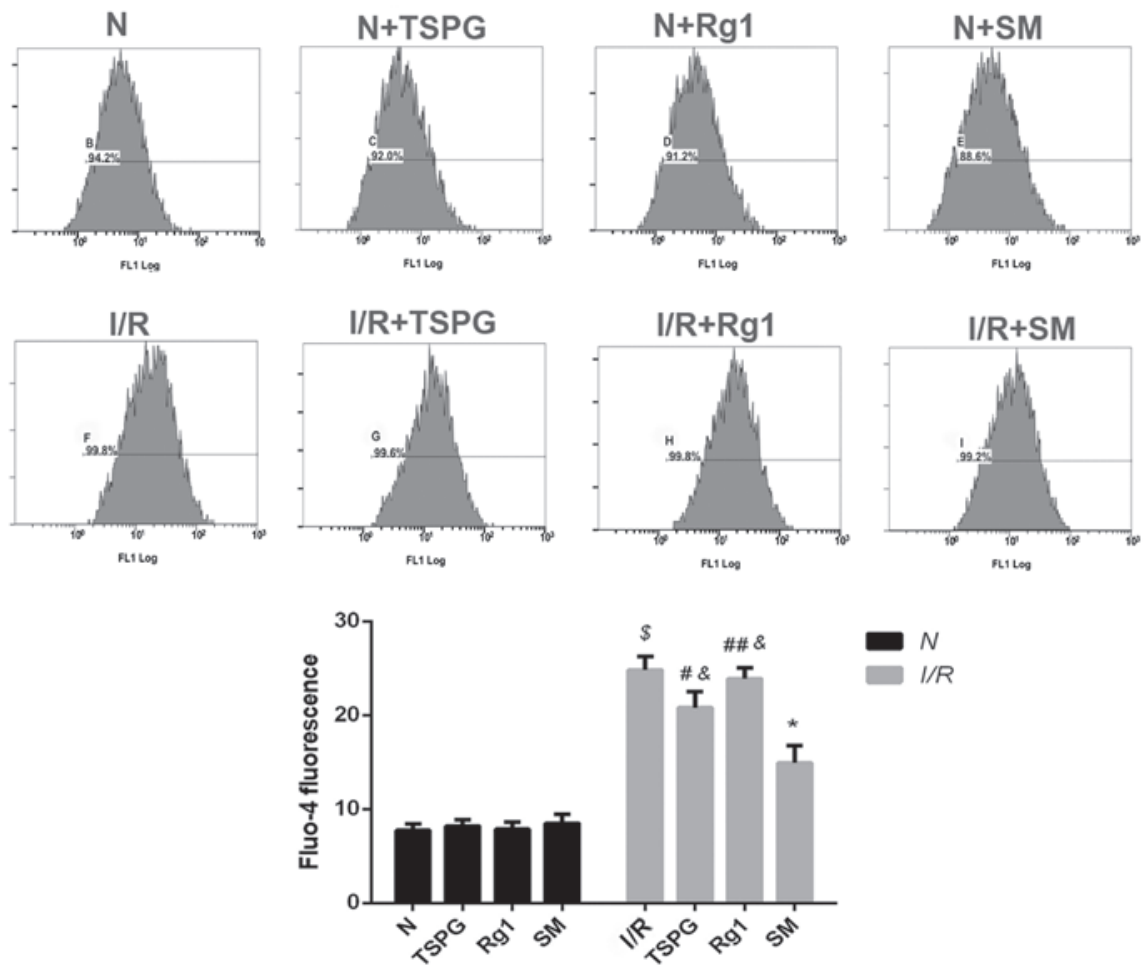

Figure 4. Effects of TSPG, Rg1 and SM on the intracellular $\mathrm{Ca}^{2+}$ concentration in cardiomyocytes following I/R. Data are expressed as the mean \pm standard deviation ( $\mathrm{n}=3) .{ }^{\$} \mathrm{P}<0.01$ vs. $\mathrm{N} ;{ }^{\&} \mathrm{P}<0.01$ vs. $\mathrm{I} / \mathrm{R}+\mathrm{SM}$; ${ }^{\mathrm{P}} \mathrm{P}<0.01$ and ${ }^{\#} \mathrm{P}<0.05$ vs. I/R; ${ }^{* *} \mathrm{P}<0.01$ and ${ }^{\# \#} \mathrm{P}<0.05$ vs. I/R + TSPG. N, normoxia; TSPG, total saponins of Panax ginseng; Rg1, ginsenoside Rg1; SM, Shenmai injection; I/R, ischemia/reperfusion. 
cantly following I/R, indicating that I/R injury successfully occurred (28).

In order to further clarify the effects of SM on myocardial cells, three different drug treatments were employed to determine their comparative efficacies in abating I/R-related complications. Ginsenoside $\mathrm{Rg} 1$ is a major bioactive component of TSPG, while TSPG is the primary bioactive component of SM. It has been established that $1 \mathrm{ml} \mathrm{SM}$ contains $65 \pm 6.68 \mu \mathrm{g}$ Rg1 and 293.38 $\pm 40.54 \mu \mathrm{g}$ TSPG (29). In accordance with similar studies concerning drug treatment of cardiomyocytes $(30,31)$, $5 \mathrm{ml} / \mathrm{l} \mathrm{SM}$ was administered in the present treatment regimen, which is equivalent to $\sim 1.5 \mathrm{mg} / 1 \mathrm{TSPG}$ and $\sim 0.325 \mathrm{mg} / \mathrm{l} \mathrm{Rg} 1$. PLB, the primary regulator of SERCA, is a small transmembrane SR protein present in the ventricles of the heart and, to a lesser extent, in the atria (32). Significant alterations in the stoichiometry between SERCA and PLB have been associated with chronic heart failure. PLB therefore functions as a physiological brake on excitation-contraction coupling, therein modulating cardiac function. The present results indicated that the reduced p-PLB expression following I/R was restored to normal levels following SM treatment, but not following treatment with TSPG or Rg1. This observation supports the conclusion that the ability of SM to counteract the negative effects of $\mathrm{I} / \mathrm{R}$ treatment is partially attributable to increased PLB phosphorylation, but not to TSPG or Rg1 when administered at similar concentrations.

The SR Ca ${ }^{2+}$-ATPase is responsible for restoring the $\mathrm{SR} \mathrm{Ca}^{2+}$ load per excitation-contraction cycle. A reduction in SERCA content is associated with reduced $\mathrm{SR} \mathrm{Ca}^{2+}$ loading and elevated $\left[\mathrm{Ca}^{2+}\right]_{\mathrm{i}}(33,34)$. The restoration of SERCA levels is potentially a critical factor in normalizing $\mathrm{Ca}^{2+}$ uptake, as observed in line-scan and frequency-dependent experiments (35). It is therefore plausible that the observed reduction in SERCA expression may partially explain the observed $\left[\mathrm{Ca}^{2+}\right]_{\mathrm{i}}$ overload in cardiomyocytes following I/R treatment. The present study has demonstrated that SM and TSPG are able to increase SERCA expression and that this improves intracellular $\mathrm{Ca}^{2+}$ cycling. Notably, the numerous changes observed following $\mathrm{I} / \mathrm{R}$ treatment were not reversed by $\mathrm{Rg} 1$ treatment, which is inconsistent with the results of previous studies $(25,26)$. This discrepancy may be attributable to the different doses of $\mathrm{Rg} 1$ used, as our study utilized a dose far below proven efficacy. To the best of our knowledge, the present study is the first to conduct a comparison between the effects of SM and its purified constituent compounds on the expression of p-PLB. Notably, the ratio of $\mathrm{p}-\mathrm{PLB} / \mathrm{PLB}$ levels was significantly reduced in the I/R group relative to that in the control $\mathrm{N}$ group $(\mathrm{P}<0.01)$; therefore, despite PLB expression remaining unaltered following I/R treatment, p-PLB expression decreased significantly.

In conclusion, the myocardial protective effects of SM following I/R treatment may be partially attributable to the effects of SM on intracellular $\mathrm{Ca}^{2+}$ homeostasis, specifically the relief of PLB inhibition. Notably, the present results indicate that the pharmacodynamic activity of TPSG is significantly superior to that of Rg1; however, the effects of SM are significantly superior to those of TSPG and Rg1.

\section{Acknowledgements}

This study was supported by grants from the National Natural Science Foundation of China (nos. 30801213 and 81170167), the International Collaboration Projects of Science and Technology Department of Zhejiang Province (no. 2011c14027) and the Foundation from Zhejiang Provincial Administration of Traditional Chinese Medicine (no. 2011ZQ013). The corresponding author is sponsored by the Zhejiang Provincial Program for the Cultivation of High-level Innovative Health talents.

\section{References}

1. World Health Oganization: Fact Sheet No. 310: The top 10 causes of death. http://www.who.int/mediacentre/factsheets/fs310/en/ Accessed November 19, 2014.

2. Braunwald E: Heart failure. JACC Heart Fail 1: 1-20, 2013.

3. Marks AR: Cardiac intracellular calcium release channels: Role in heart failure. Circ Res 87: 8-11, 2000.

4. Ramón de Berrazueta J: The role of calcium in the regulation of normal vascular tone and in arterial hypertension. Rev Esp Cardiol (52 Suppl) 3: 25-33, 1999 (In Spanish).

5. Spinale FG, de Gasparo M, Whitebread S, Hebbar L, Clair MJ, Melton DM, Krombach RS, Mukherjee R, Iannini JP and O SJ: Modulation of the renin-angiotensin pathway through enzyme inhibition and specific receptor blockade in pacing-induced heart failure: I. Effects on left ventricular performance and neurohormonal systems. Circulation 96: 2385-2396, 1997.

6. Kranias EG and Hajjar RJ: Modulation of cardiac contractility by the phospholamban/SERCA2a regulatome. Circ Res 110: 1646-1660, 2012.

7. Sun Y, Hu S, Wang L, Hu Y and Zhou J: Comparison of low and high doses of carvedilol on restoration of cardiac function and calcium-handling proteins in rat failing heart. Clin Exp Pharmacol Physiol 32: 553-560, 2005.

8. Sun Y, Hu S, Wang L, Hu Y and Zhou J: Changes of calcium handling protein after acute myocardial infarction in rats and effect of carvedilol. Zhong Guo Bing Li Sheng Li Za Zhi 21: 1085-1089, 2005 (In Chinese).

9. Changyu Qin and Zhizhen Qin (eds): Zheng Yin Mai Zhi. First edition. Shanghai wei sheng chu ban she, Shanghai, 1958.

10. Hou YZ, Mao JY, Wang XL, Liu CX and Zhang C: Shenmai injection in heart failure patients: A systematic review and Meta-analysis. Zhong Guo Xun Zheng Yi Xue Za Zhhi She 10: 939-945, 2010.

11. Zhang L, Wang BH, Hu J and Shang HC: Shenmai injection for children with viral myocarditis: A systematic review. Zhong Guo Xun Zheng Yi Xue Za Zhi She 10: 700-706, 2010.

12. Li KJ: Shenmai injection for acute ischemic stroke: A systematic review of randomized controlled trial. Zhong Yi Yao Xue Bao 34: 4-7, 2006

13. Liu JG: Systematic review on Shenmai Injection treatment of dilated cardiomyopathy. Zhong Cheng Yao 34: 1456-1461, 2012.

14. Hu J, Zhang W, Xie YM, Wang LX, Nie XL and Zhang YL: Meta-analysis of Shenmai injection treatment for acute myocardial infarction. Zhong Guo Zhong Yao Za Zhi She 37: 2760-2767, 2012.

15. Gong W and Hu Z: Clinical application of Shenmai injection. Yi Yao Dao Bao 19: 181-183, 2000 (In Chinese).

16. Zhang L, Hu J, Xiao L, Zhang Y, Zhao W, Zheng W and Shang H: Adverse drug reactions of Shenmai injection: A systematic review. J Evid-Based Med 3: 177-182, 2010.

17. Bai CX, Takahashi K, Masumiya H, Sawanobori $\mathrm{T}$ and Furukawa T: Nitric oxide-dependent modulation of the delayed rectifier $\mathrm{K}^{+}$current and the $\mathrm{L}$-type $\mathrm{Ca}^{2+}$ current by ginsenoside $\mathrm{Re}$, an ingredient of Panax ginseng, in guinea-pig cardiomyocytes. Br J Pharmacol 142: 567-575, 2004.

18. Scott GI, Colligan PB, Ren BH and Ren J: Ginsenosides Rb1 and Re decrease cardiac contraction in adult rat ventricular myocytes: Role of nitric oxide. Br J Pharmacol 134: 1159-1165, 2001.

19. Bai CX, Sunami A, Namiki T, Sawanobori T and Furukawa T: Electrophysiological effects of ginseng and ginsenoside Re in guinea pig ventricular myocytes. Eur J Pharmacol 476: 35-44, 2003.

20. Liu Z, Li Z and Liu X: Effect of ginsenoside Re on cardiomyocyte apoptosis and expression of Bcl-2/Bax gene after ischemia and reperfusion in rats. J Huazhong Univ Sci Technolog Med Sci 22: 305-309, 2002.

21. He L, Sun S and Fan J: Effect of Shenmai injection on neurocyte apoptosis and change of cytoplasmic calcium. Zhong Guo Zhong Xi Yi Jie He Za Zhi 21: 605-607, 2001 (In Chinese). 
22. Hou M and Ao D: Study on the mechanism of ginsenosides against ischemia-reperfusion injury of myocardium. Zhong Guo Xiong Xin Xue Guan Wai Ke Lin Chuang Za Zhi 7: 256-9, 2000 (In Chinese)

23. Li Y, Cui X, Pan L, Li Z, Ji C and Du K: Electron microscopic observation and $\mathrm{Ca}^{2+}$ analysis on protective effect of ginsenoside from fruit on myocardium of hemorrhagic shock in dogs. Bai Qiu En Yi Ke Da Xue Xue Bao 24: 452-454, 1998 (In Chinese).

24. Zhao X, Li Z, Cao Y, Geng Q, An L and Tang H: (2001) The influence of ginsenosides on intracellular free calcium concentration during hypoxia in guinea pigs. J Chin Med Univ 30: 431-434, 2001.

25. He Q, Sun J, Wang Q, Wang W and He B: Neuroprotective effects of ginsenoside Rg1 against oxygen-glucose deprivation in cultured hippocampal neurons. J Chin Med Assoc 77: 142-149, 2014.

26. Zhu D, Wu L, Li CR, Wang XW, Ma YJ, Zhong ZY, Zhao HB, Cui J, Xun SF, Huang XL, et al: Ginsenoside Rg1 protects rat cardiomyocyte from hypoxia/reoxygenation oxidative injury via antioxidant and intracellular calcium homeostasis. J Cell Biochem 108: 117-124, 2009.

27. Simpson $P$ and Savion S: Differentiation of rat myocytes in single cell cultures with and without proliferating nonmyocardial cells. Cross-striations, ultrastructure and chronotropic response to isoproterenol. Circ Res 50: 101-116, 1982.

28. Stamm C, Friehs I, Cowan DB, Cao-Danh H, Choi YH, Duebener LF, McGowan FX and del Nido PJ: Dopamine treatment of postischemic contractile dysfunction rapidly induces calcium-dependent pro-apoptotic signaling. Circulation 106 : I290-I298, 2002.
29. Cao S, Nie L, Wang G and Lin R: Study on ginsenosides contents of Shenmai injection and its intermediates by UPLC. Yao Wu Fen Xi Za Zhi 7: 1264-68, 2014 (In Chinese).

30. Chen Y, Hao R and Huang Q: Shenmai injection protects rat cardiomyocytes from angiotensin II-induced apoptosis in vitro. Zhong Guo Bing Li Sheng Li Za Zhi 9: 76-80, 2004 (In Chinese).

31. Hao R, Lou J, Zhang Y, Zheng H and Huang Q: The effect of Shenmai injection on cardiac myocyte apoptosis after hypoxia. Zhong Guo Bing Li Sheng Li Za Zhi 4: 660-663, 2007 (In Chinese).

32. Koss KL, Ponniah S, Jones WK, Grupp IL and Kranias EG: Differential phospholamban gene expression in murine cardiac compartments. Molecular and physiological analyses. Circ Res 77: 342-353, 1995

33. Ito K, Yan X, Feng X, Manning WJ, Dillmann WH and Lorell BH: Transgenic expression of sarcoplasmic reticulum $\mathrm{Ca}(2+)$ ATPase modifies the transition from hypertrophy to early heart failure. Circ Res 89: 422-429, 2001

34. Schmidt U, del Monte F, Miyamoto MI, Matsui T, Gwathmey JK, Rosenzweig A and Hajjar RJ: Restoration of diastolic function in senescent rat hearts through adenoviral gene transfer of sarcoplasmic reticulum $\mathrm{Ca}(2+)-A T P a s e$. Circulation 101: 790-796, 2000.

35. Plank DM, Yatani A, Ritsu H, Witt S, Glascock B, Lalli MJ, Periasamy M, Fiset C, Benkusky N, Valdivia $\mathrm{HH}$ and Sussman MA: Calcium dynamics in the failing heart: Restoration by beta-adrenergic receptor blockade. Am J Physiol Heart Circ Physiol 285: H305-H315, 2003. 